\title{
Synthesis of Hollow Silica Nanospheres by Sacrificial Polystyrene Templates for Thermal Insulation Applications
}

\author{
Linn Ingunn C. Sandberg, ${ }^{1}$ Tao Gao, ${ }^{2}$ Bjørn Petter Jelle, ${ }^{1,3}$ and Arild Gustavsen ${ }^{2}$ \\ ${ }^{1}$ Department of Civil and Transport Engineering, Norwegian University of Science and Technology (NTNU), \\ 7491 Trondheim, Norway \\ ${ }^{2}$ Department of Architectural Design, History and Technology, Norwegian University of Science and Technology (NTNU), \\ 7491 Trondheim, Norway \\ ${ }^{3}$ Department of Materials and Structures, SINTEF Building and Infrastructure, 7465 Trondheim, Norway
}

Correspondence should be addressed to Linn Ingunn C. Sandberg; linn.sandberg@ntnu.no

Received 10 January 2013; Accepted 21 February 2013

Academic Editor: Marcel Ausloos

Copyright (C) 2013 Linn Ingunn C. Sandberg et al. This is an open access article distributed under the Creative Commons Attribution License, which permits unrestricted use, distribution, and reproduction in any medium, provided the original work is properly cited.

\begin{abstract}
Monodisperse polystyrene (PS) spheres with controllable size have been synthesized by a straight forward and simple procedure. The as-synthesized PS spheres have a typical diameter ranging from $\sim 180 \mathrm{~nm}$ to $\sim 900 \mathrm{~nm}$, where a reduced sphere size is obtained by increasing the polyvinylpyrrolidone (PVP)/styrene weight ratio. The PS spheres function as sacrificial templates for the fabrication of hollow silica nanospheres (HSNSs) for thermal insulation applications. By modifying the silica coating process, HSNSs with different surface roughness are obtained. All resulting HSNSs show typically a thermal conductivity of about $20 \mathrm{~mW} /(\mathrm{mK})$, indicating that the surface phonon scattering is probably not significant in these HSNS samples.
\end{abstract}

\section{Introduction}

A substantial amount of the total heat loss of residential buildings passes through the opaque building envelopes, as these components normally account for the largest contact area towards the outside environment. As the demand for energy efficiency in building codes becomes increasingly stringent, it is necessary to reduce the unwanted thermal loss of existing and future building envelopes. This can either be done by increasing the wall thickness when conventional thermal insulation materials such as mineral wool or expanded polystyrene (EPS) is used, or install state-of-theart superinsulating materials such as aerogel blankets/mats or vacuum insulation panels (VIPs). Increased wall thickness is unwanted as floor space is lost and/or modification of the adjoining building elements becomes necessary. Major downsides with state-of-the-art insulation materials of today are their high cost (VIP and aerogel), their fragility, risk of puncturing, no building site adaptation, and ageing issues (VIP).
An alternative to meet the demands of the future regarding thermal resistance of building envelopes is to develop a new generation of thermal insulation materials. One path which can be followed, is to create nano insulation materials (NIMs), which aim to utilize physical principles such as the Knudsen effect to reduce the thermal conductivity of the material to a minimum [1]. A NIM is a homogeneous, nanostructured material with closed or open nanosized pores where the gas molecules in the pores are more likely to collide with the pore walls rather than with each other, see Figure 1 [2]. The overall property of NIM can be controlled by tuning, for example, the pore sizes (i.e., $D$ or $L$ in Figure 1), the chemical composition of the matrix materials, and the packing manner/density. For example, Luo and Ye have reported recently a nanofoam consisting of polymer nanocapsules [3], of which the thermal conductivity is about $0.016-0.023 \mathrm{~W} /(\mathrm{mK})$, depending on the sizes of the nanocapsules. Similar results have also been reported for inorganic materials such as hollow silica nanospheres (HSNSs) $[2,4,5]$. 


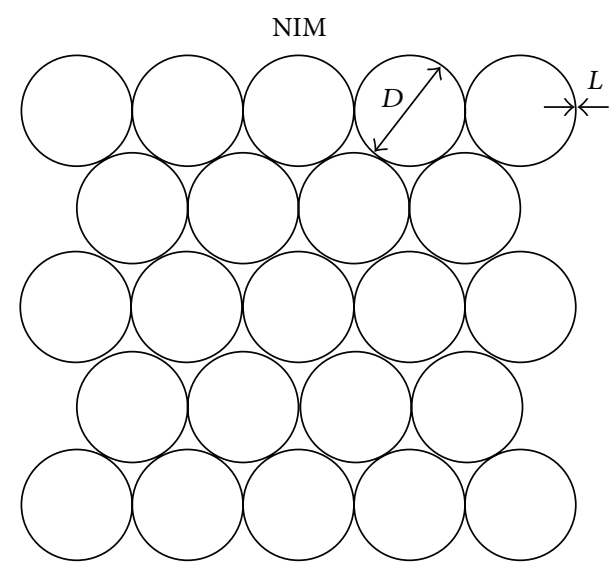

(a)

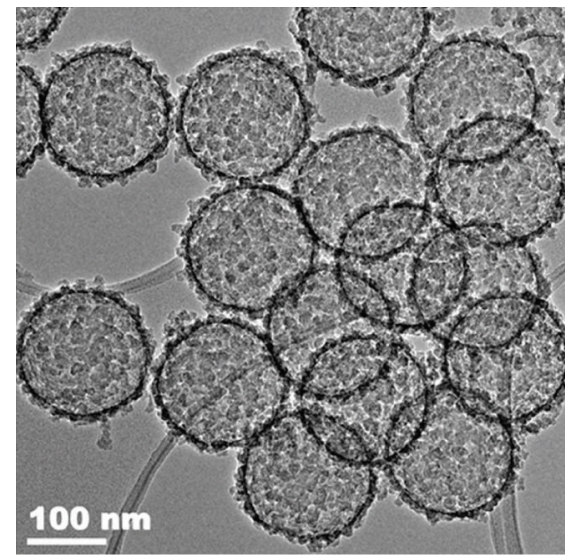

(b)

Figure 1: Conceptual model of a hollow nanosphere NIM with a description of the pore size $D$ and shell thickness $L$ (a) and a representative TEM image of hollow silica nanospheres (b) [2].

The Knudsen equation relating the mean free path of the gas molecules and the average diameter of the pores may be written as

$$
\lambda_{\mathrm{gas}}=\frac{\lambda_{\mathrm{gas}, 0}}{1+2 \beta \mathrm{Kn}},
$$

where

$$
\mathrm{Kn}=\frac{\sigma_{\text {mean }}}{\delta}=\frac{k_{B} T}{\sqrt{2} \pi d^{2} p \delta},
$$

where $\lambda_{\text {gas }}$ is the thermal conductivity of the gas inside the nanosized pores, $\lambda_{\text {gas }, 0}$ is the thermal conductivity of the gas at STP, $\beta$ is the energy transfer efficiency of the molecule-wall collision (a unit-less number between 1.5 and 2.0 ), $\mathrm{Kn}$ is the Knudsen number, $\sigma_{\text {mean }}$ is the mean free path of the gas molecules $(\mathrm{m}), \delta$ is the characteristic pore size of the material $(\mathrm{m}), d$ is the collision diameter of the gas molecules $(\mathrm{m}), p$ is the gas pressure inside the pores $(\mathrm{Pa})$, and $T$ is the temperature $(\mathrm{K})$. It is evident that for pores with diameters of a few nanometers, the Knudsen number becomes very large, resulting eventually in $\lambda_{\text {gas }} \rightarrow 0$. Taking HSNSs with pore sizes of about $100 \mathrm{~nm}$ in dimension as an example, a reduced gaseous thermal conductivity $\lambda_{\text {gas }}$ of about $0.008 \mathrm{~W} /(\mathrm{mK})$ can be obtained, compared to the thermal conductivity value of $0.026 \mathrm{~W} /(\mathrm{mK})$ for air at STP.

The objective of this work is to attempt to make a NIM from HSNSs. There are several parameters which may be important to determine the overall properties of HSNS NIMs, for example, the dimensions of the pore and the thickness of the silica shell. Moreover, the surface roughness of the HSNSs is likely to have an effect on the thermal conductivity of the final product. For example, research on thermoelectric materials has shown that the thermal conductivity of materials is reduced with an increased amount of phonon scattering within the material $[6,7]$. It is therefore reasonable to assume that a shell built up from many small silica nanoparticles has a higher degree of phonon scattering, and thus a lower thermal conductivity, compared to a shell consisting of larger silica nanoparticles.

\section{Experimental}

2.1. Materials. Reagent grade styrene (St), polyvinylpyrrolidone (PVP, molecular weight $\approx 40000$ ), potassium persulfate (KPS), tetraethyl orthosilicate (TEOS), ammonium hydroxide solution $\left(\mathrm{NH}_{4} \mathrm{OH}, 28-30 \mathrm{wt} \%\right)$, absolute ethanol (EtOH, $>99.8 \%$ ), and $96 \%$ ethanol were received from Sigma-Aldrich and used without further purification. Distilled water was used throughout the synthesis.

\subsection{Preparation of PVP-Stabilized Polystyrene Templates with} Varying Size. $10 \mathrm{~g}$ of styrene and a given amount of PVP were added to $90 \mathrm{~g}$ of distilled water in a $250 \mathrm{~mL}$ Erlenmeyer flask. The mixture was magnetically stirred at $300 \mathrm{rpm}$ for $15 \mathrm{~min}$. Thereafter, a diluted KPS aqueous solution ( $0.10 \mathrm{~g}$ KPS in $10 \mathrm{~g}$ water) was added to the styrene $/ \mathrm{H}_{2} \mathrm{O}$ mixture before the flask was immersed in an oil bath. The temperature of the oil bath was gradually increased to $70^{\circ} \mathrm{C}$. The solution was left at this temperature for $\sim 24$ hours, after which it was cooled down to room temperature.

2.3. Coating the Polystyrene Templates with Silica. $190 \mathrm{~g}$ of $\mathrm{EtOH}(100 \%$ and/or $96 \%)$ and $12 \mathrm{~g}$ of polystyrene templates in suspension (created with a $\mathrm{PVP} /$ styrene weight ratio of 0.10 , see also Table 1 ) were magnetically stirred for $15 \mathrm{~min}$ before $3 \mathrm{~mL}$ of $\mathrm{NH}_{4} \mathrm{OH}$ was added. $20 \mathrm{~mL}$ of TEOS solution $(10 \mathrm{~mL}$ of TEOS in $10 \mathrm{~mL}$ of $96 \% \mathrm{EtOH})$ was used for each coating procedure. By varying the TEOS concentration during the reaction, four different samples were obtained for comparison as follows:

Sample A: TEOS solution added into a 100\% EtOH/PS solution;

Sample B: TEOS solution added into a 96\% EtOH/PS solution; 


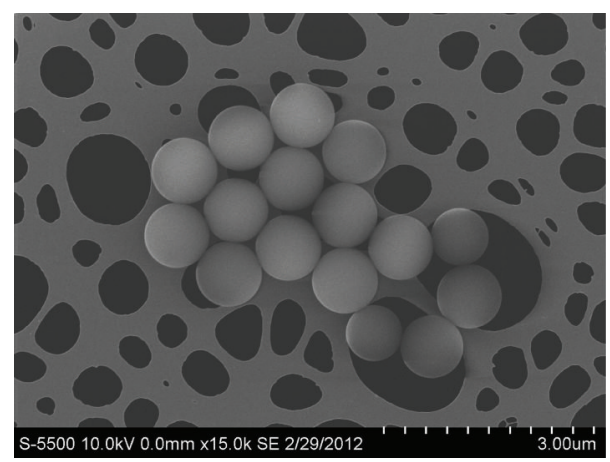

(a)

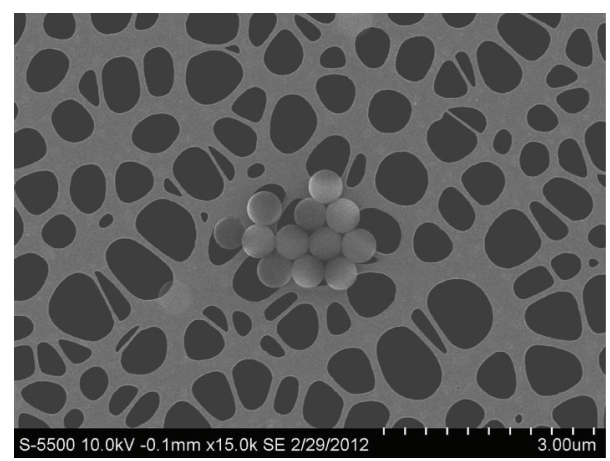

(b)

FIGURE 2: SEM images of polystyrene templates created with a $\mathrm{PVP} /$ styrene-ratio of 0.005 (a) and 0.0075 (b), $15 \mathrm{k}$ magnification.

Sample C: TEOS solution added (5 times, $4 \mathrm{~mL}$ each time) into a $96 \% \mathrm{EtOH} / \mathrm{PS}$ solution;

Sample D: TEOS solution added (100 times, $0.2 \mathrm{~mL}$ each time) into a $96 \% \mathrm{EtOH} / \mathrm{PS}$ solution.

Here, the concentration of TEOS in Sample A was high in the beginning; for Sample D, the concentration of TEOS was lower initially.

The silica-coated PS templates were extracted by centrifugation at $8000 \mathrm{rpm}$ for $10 \mathrm{~min}$. The samples were left to airdry overnight before the polystyrene cores were removed by annealing at $500^{\circ} \mathrm{C}$ for 5 hours in air.

2.4. Characterization. The size and morphology of the assynthesized materials were characterized with a Hitachi S5500 scanning electron microscope (SEM), which was also equipped with a transmission mode. A small sample of the solution containing the particles was dripped onto a TEM grid and dried naturally in air. The particles were studied with secondary electrons and an acceleration voltage of $10 \mathrm{kV}$ and a current of $7 \mu \mathrm{A}$ was set as a standard, whereas $30 \mathrm{kV}$ was used in bright field transmission mode.

The thermal conductivity of the resulting materials was measured on a Hot Disk TPS 2500S Thermal Constants Analyser. As the amount of sample material was scarce, a small sensor with a diameter of $3.189 \mathrm{~mm}$ was utilized.
TABLE 1: Average diameter of the polystyrene templates by varying the PVP/styrene-ratio

\begin{tabular}{lc}
\hline PVP/styrene-ratio & Average diameter $(\mathrm{nm})^{*}$ \\
\hline 0.0050 & $900 \pm 30$ \\
0.0075 & $475 \pm 4$ \\
0.010 & $355 \pm 6$ \\
0.050 & $281 \pm 1$ \\
0.10 & $228 \pm 1$ \\
0.15 & $178 \pm 1$ \\
\hline
\end{tabular}

${ }^{*}$ The uncertainties are given as two times the standard deviation of the mean, that is, with a confidence interval of $95.45 \%$.

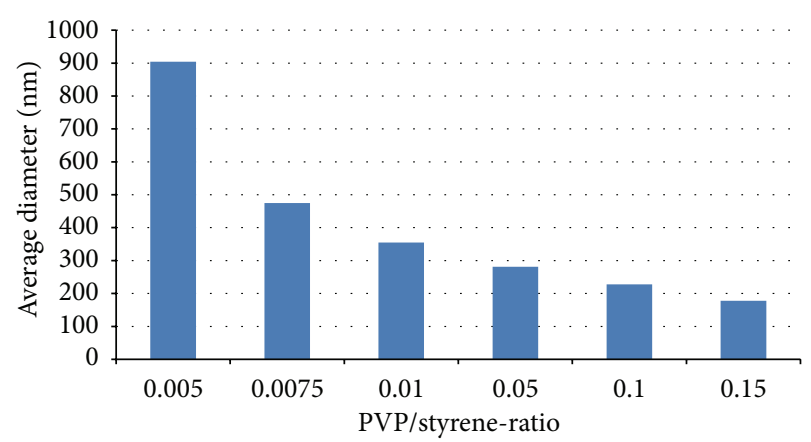

FIGURE 3: Average diameter of the polystyrene templates as a function of PVP/styrene-ratio.

\section{Results and Discussion}

There are several techniques which may be employed to create monodisperse PS spheres, one of which is emulsifierfree emulsion polymerization [8]. Several reports have been published where, for example, the initiator and stabilizer type and concentration, and the styrene concentration and the reaction temperature, have been varied [9-11]. These parameters have an effect on both the size and the monodispersity of the resulting PS spheres.

The PVP/styrene-ratio had a prominent effect on both the resulting average size and the degree of monodispersity of the PS spheres $[9,11]$. In this work, it is found that the effect of varying the $\mathrm{PVP} /$ styrene-ratio was largest when the ratio was small; that is, the largest change in PS particle size was observed at $\mathrm{PVP} /$ styrene-ratio close to zero. Here, a $50 \%$ increase in the amount of stabilizer (from $0.05 \mathrm{~g}$ PVP to $0.075 \mathrm{~g}$ ) leads to a halving of the resulting particle size (from 900 to $475 \mathrm{~nm}$, see Figure 2), while a 50\% increase from, for example, $1 \mathrm{~g}$ to $1.5 \mathrm{~g}$ decreased the average particle size by only $\sim 20 \%$. The results of controlling the size of the polystyrene templates by varying the $\mathrm{PVP} /$ styrene weight ratio are presented in Table 1 .

Hence, the relationship between PVP/styrene-ratio and the average PS particle size is not linear in nature, as can be depicted from Figure 3.

Interestingly, silica nanoparticles do not stick to polystyrene templates made with a $\mathrm{PVP} /$ styrene-ratio of 0.0075 , but 


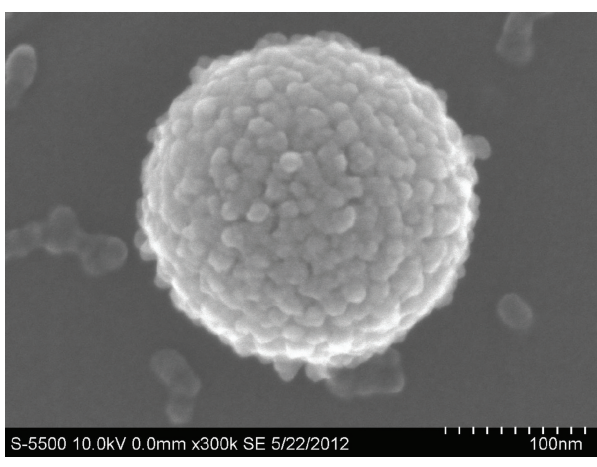

(a)

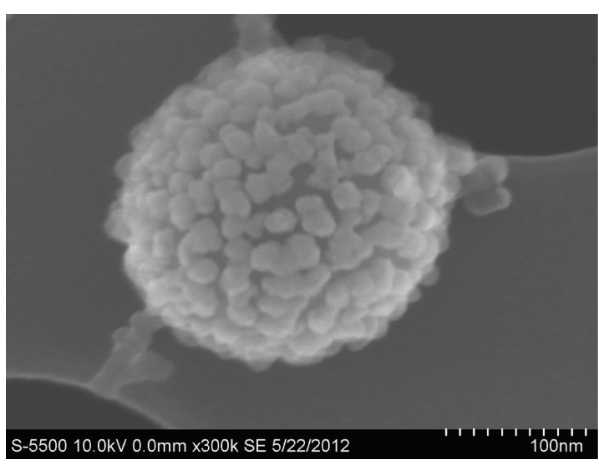

(c)

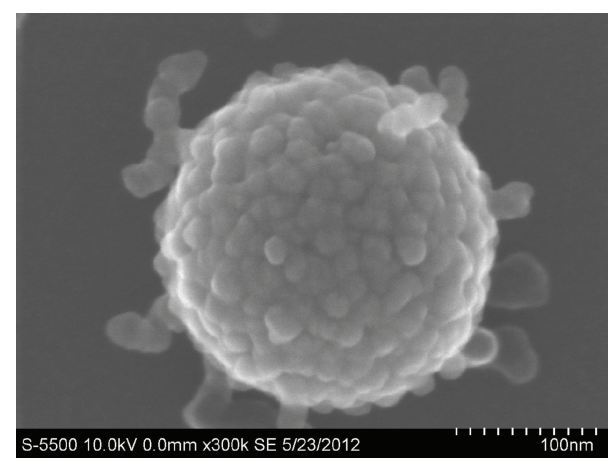

(b)

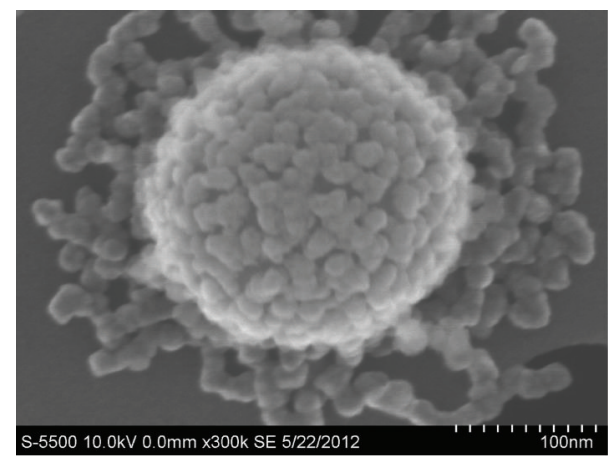

(d)

Figure 4: SEM images of (a) for Sample A, (b) for Sample B, (c) for Sample C, and (d) for Sample D. The PS template is within the spheres.

they do attach when the ratio is increased to 0.05. It indicates the role of surface absorbed PVP molecules on attracting primary silica particles from the solution. When the coverage of PVP is not sufficient, that is, at low PVP/styrene-ratio, their buffering effect is not strong enough to mask the negative charge of the PS particles, which is due to the presence of sulfate groups [11]. Hence, the negatively charged silica particles are repelled from the template and do not cover the polystyrene particles. In this regard, polystyrene templates with a positively charged surface seem interesting. Ongoing research is carried out by mixing styrene with various comonomers to create positively charged PS templates.

Experiments were also carried out to investigate if the surface roughness of the silica coating could be controlled, and if varying the surface roughness would affect the resulting bulk thermal conductivity of the final products. The amount of water in the synthesis did have a small effect on the size of the silica nanoparticles coating the polystyrene templates, and hence, the surface roughness of the hollow silica spheres. This may be explained by how presence of water is vital for hydrolysis of TEOS to take place. If less water is present, the hydrolysis reaction runs slower and smaller silica particles stick to the PS template before they are allowed to grow any larger. It was also assumed that the concentration of TEOS had a certain effect, but this showed to be of less importance. The appearance of the silica-coated polystyrene templates did not differ to a great extent, which is demonstrated in Figure 4. In general, the surface roughness of Sample B, C, and D is quite similar, whereas the silica nanoparticles constituting
TABLE 2: Average thermal conductivity of the four samples created by various coating procedures.

\begin{tabular}{lc}
\hline Sample name & Average thermal conductivity $(\mathrm{mW} /(\mathrm{mK}))^{*}$ \\
\hline A & $21 \pm 3$ \\
B & $21 \pm 1$ \\
C & $23 \pm 2$ \\
D & $20 \pm 2$ \\
\hline
\end{tabular}

*The uncertainties are given as two times the standard deviation of the mean, that is, with a confidence interval of $95.45 \%$.

the shell of the hollow silica sphere in Sample A are slightly smaller as the amount of water present has been restricted.

The SEM photos in Figure 5 verify that none of the four samples collapsed during the heat treatment when burning off the PS polymer core at $500^{\circ} \mathrm{C}$.

It should be noted that a large variation of thermal conductivity values is obtained both within one set of testing procedure and between different testing procedures. Hence, the results of various combinations of heating power and measurement time with the Hot Disk apparatus were analyzed and an average of the most trustworthy conductivity values was calculated. What could be considered as a trustworthy conductivity was one that met all the requirements set by the Hot Disk instrument manufacturer. The average thermal conductivities of the four samples with corresponding uncertainties are shown in Table 2, depicting similar thermal 


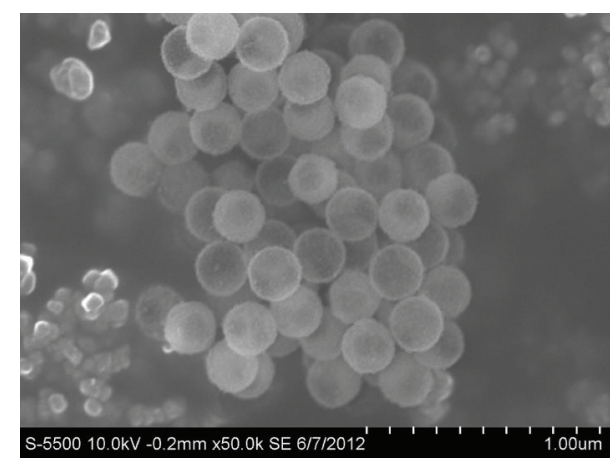

(a)

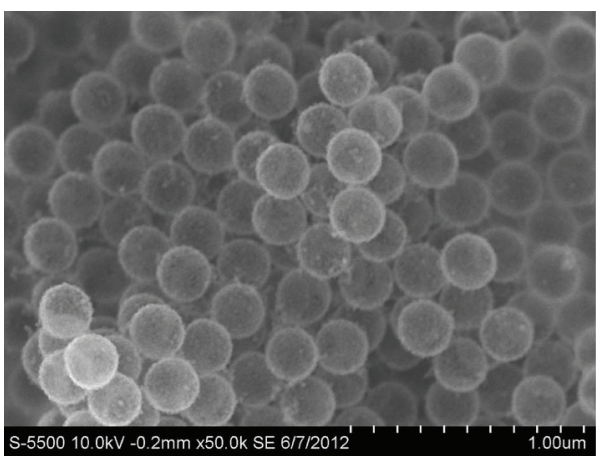

(c)

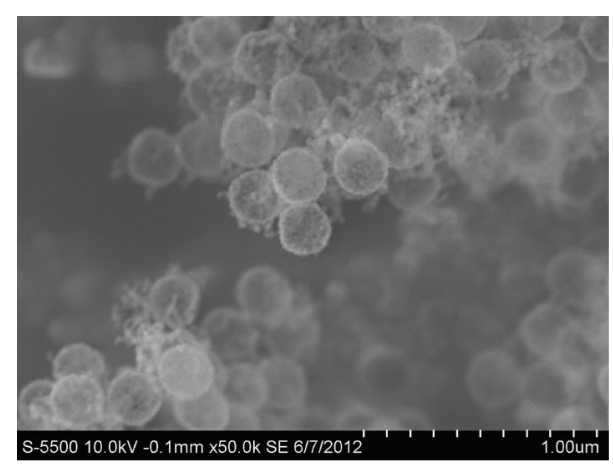

(b)

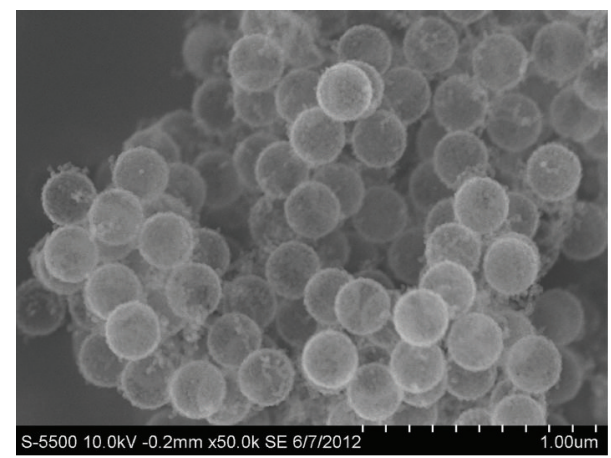

(d)

Figure 5: SEM images of (a) for Sample A, (b) for Sample B, (c) for Sample C, and (d) for Sample D after removal of PS templates.

conductivity values for all four samples within an uncertainty of two times the standard deviation of the mean, that is, with a confidence interval of $95.45 \%$.

\section{Conclusions}

The size of the polystyrene (PS) templates could easily be controlled by adjusting the $\mathrm{PVP} /$ styrene weight ratio. As the polystyrene spheres and the silica nanoparticles both are negatively charged, there is a minimum amount of stabilizer (PVP) which is necessary for the silica nanoparticles to stick to the PS template. The minimum ratio lies between 0.0075 and 0.05 , as PS templates created by a PVP/styrene-ratio of 0.0075 do not attract silica nanoparticles and those created by a ratio of $0.05 \mathrm{do}$. It is likely that the surface roughness of the hollow silica spheres can be controlled by varying the amount of water in the synthesis solution and should hence be subject to further studies.

\section{Acknowledgments}

This work has been supported by the Research Council of Norway and several partners through the NTNU and SINTEF "Research Centre on Zero Emission Buildings" (ZEB).

\section{References}

[1] B. P. Jelle, A. Gustavsen, and R. Baetens, "The path to the high performance thermal building insulation materials and solutions of tomorrow," Journal of Building Physics, vol. 34, no. 2, pp. 99-123, 2010.

[2] T. Gao, L. I. C. Sandberg, B. P. Jelle, and A. Gustavsen, "Nano insulation materials for energy efficient buildings: a case study on hollow silica nanospheres," in Fuelling the Future: Advances in Science and Technologies for Energy Generation, Transmission and Storage, A. Mendez-Vilas, Ed., pp. 535-539, BrownWalker, Boca Raton, Fla, USA, 2012.

[3] Y. Luo and C. Ye, "Using nanocapsules as building blocks to fabricate organic polymer nanofoam with ultra low thermal conductivity and high mechanical strength," Polymer, vol. 53, pp. 5699-5705, 2012.

[4] Q. Yue, Y. Li, M. Kong et al., "Ultralow density, hollow silica foams produced through interfacial reaction and their exceptional properties for environmental and energy applications," Journal of Materials Chemistry, vol. 21, no. 32, pp. 12041-12046, 2011.

[5] Y. Liao, X. Wu, H. Liu, and Y. Chen, "Thermal conductivity of powder silica hollow spheres," Thermochimica Acta, vol. 526, pp. 178-184, 2011.

[6] J. He, J. R. Sootsman, S. N. Girard et al., "On the origin of increased phonon scattering in nanostructured $\mathrm{PbTe}$ based thermoelectric materials," Journal of the American Chemical Society, vol. 132, no. 25, pp. 8669-8675, 2010.

[7] S. K. Bux, J. P. Fleurial, and R. B. Kaner, "Nanostructured materials for thermoelectric applications," Chemical Communications, vol. 46, no. 44, pp. 8311-8324, 2010.

[8] J. Yang, J. U. Lind, and W. C. Trogler, "Synthesis of hollow silica and titania nanospheres," Chemistry of Materials, vol. 20, no. 9, pp. 2875-2877, 2008. 
[9] D. S. Yun, H. S. Lee, H. G. Jang, and J. W. Yoo, "Controlling size and distribution for nano-sized polystyrene spheres," Bulletin of the Korean Chemical Society, vol. 31, no. 5, pp. 1345-1348, 2010.

[10] H. Zou, S. Wu, Q. Ran, and J. Shen, "A simple and lowcost method for the preparation of monodisperse hollow silica spheres," Journal of Physical Chemistry C, vol. 112, no. 31, pp. 11623-11629, 2008.

[11] X. Du and J. He, "Facile size-controllable syntheses of highly monodisperse polystyrene nano- and microspheres by polyvinylpyrrolidone-mediated emulsifier-free emulsion polymerization," Journal of Applied Polymer Science, vol. 108, no. 3, pp. 1755-1760, 2008. 

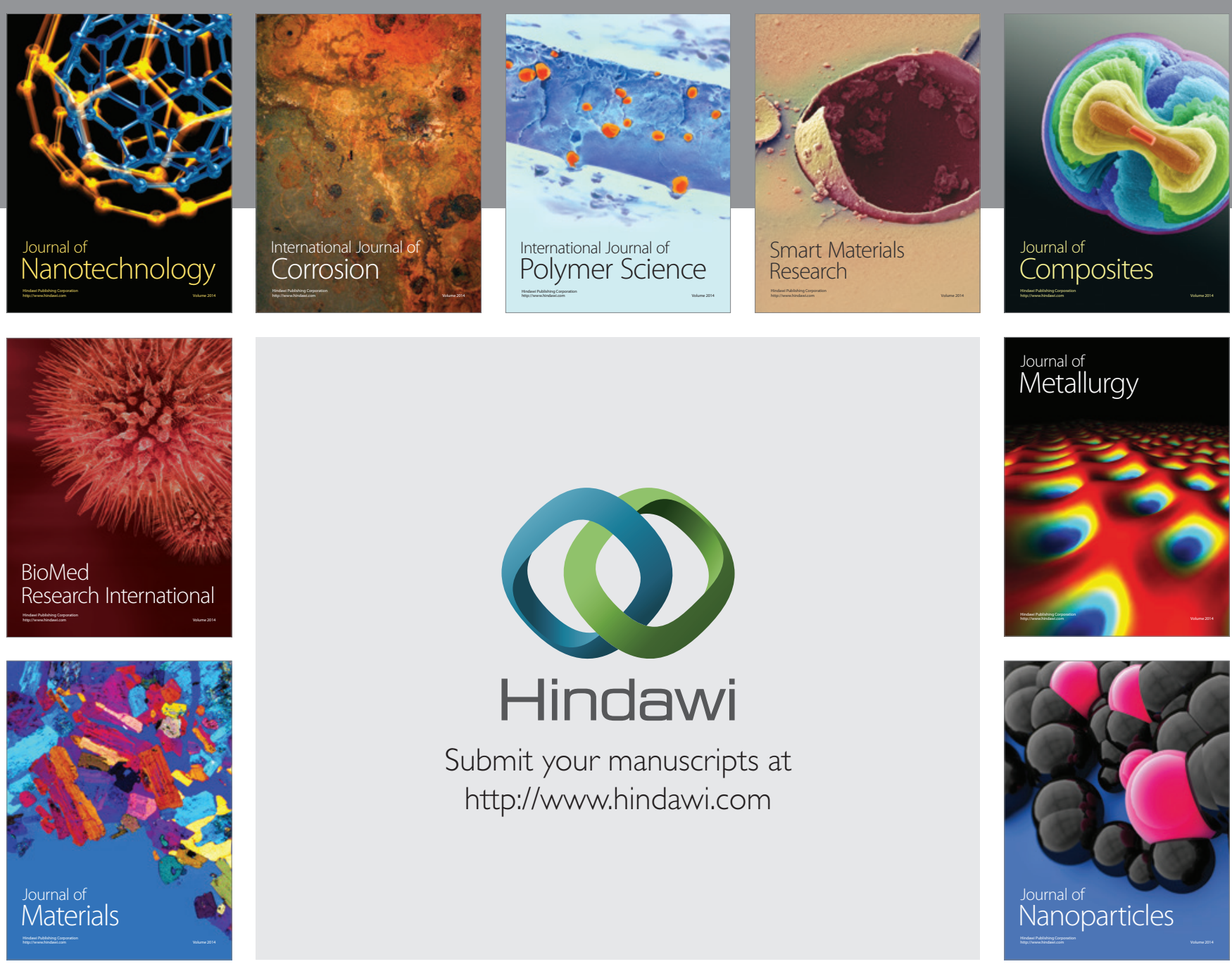

Submit your manuscripts at http://www.hindawi.com
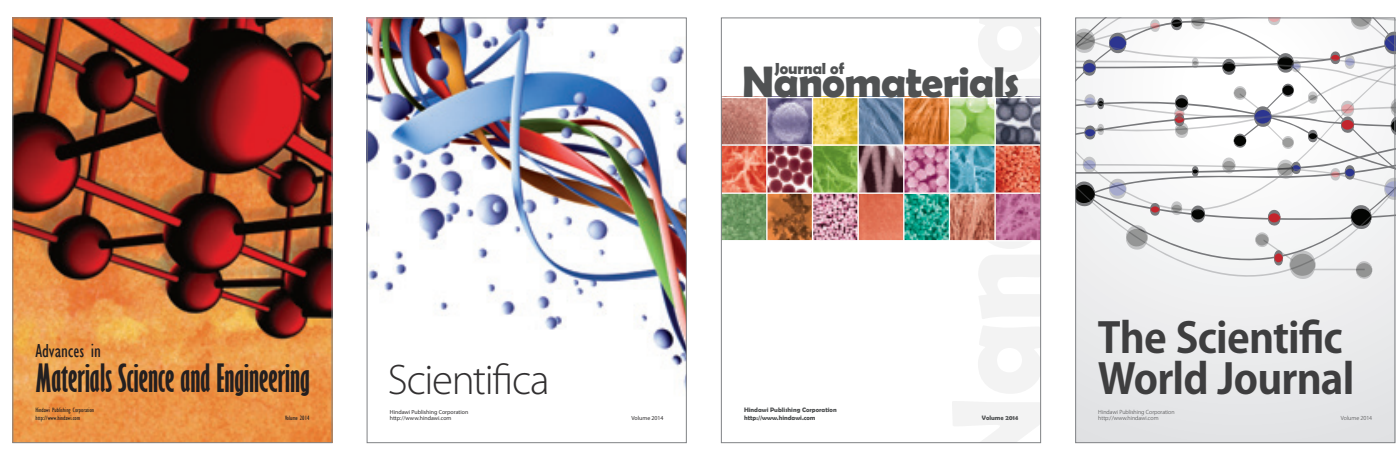

\section{The Scientific World Journal}
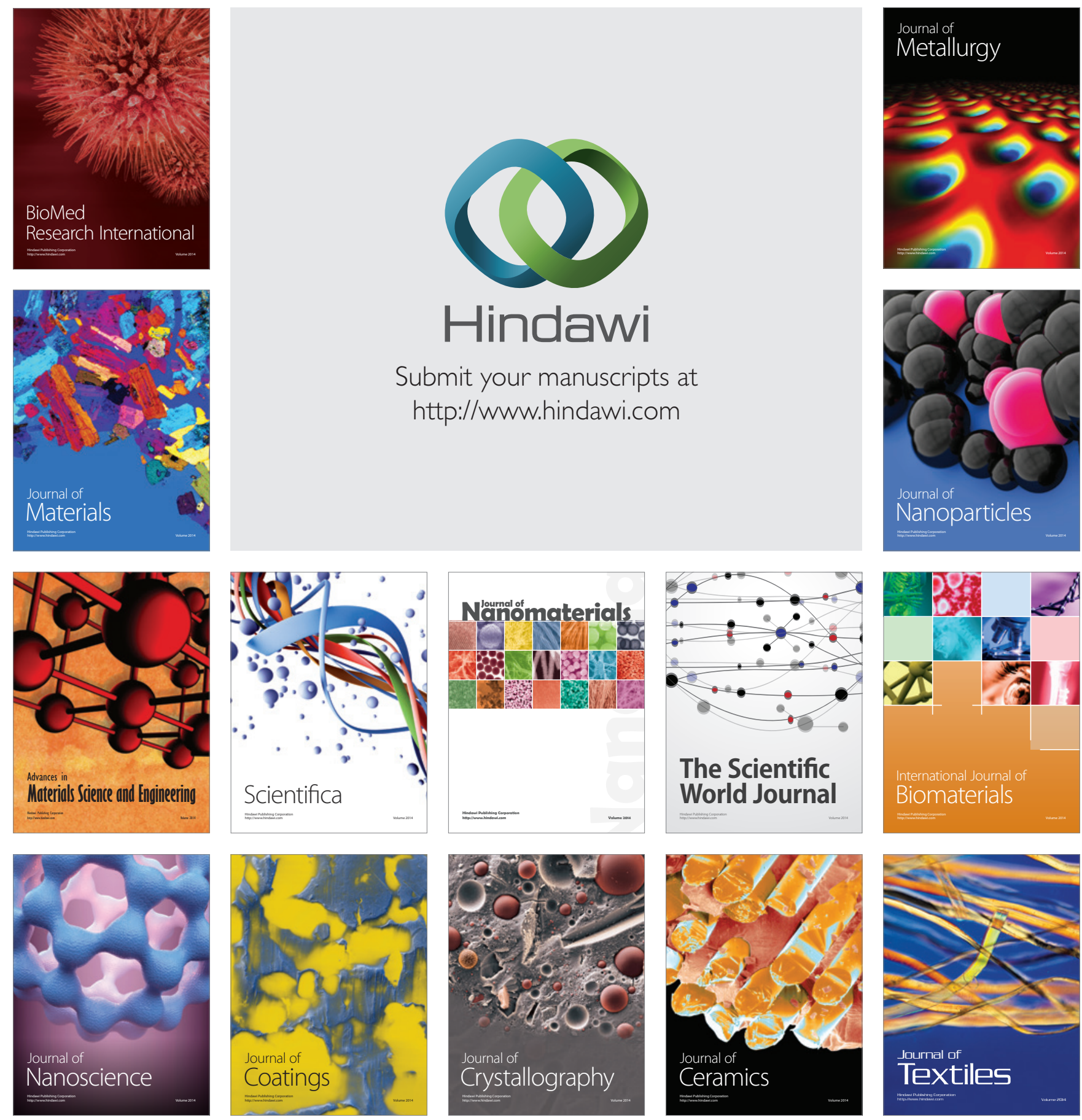